\title{
A NOTE ON PARALLEL DISTINGUISHABILITY OF TWO QUANTUM OPERATIONS*
}

\author{
CHI-KWONG LI ${ }^{\dagger}$, YUE LIU ${ }^{\ddagger}$, CHAO MA ${ }^{\S}$, AND DIANE CHRISTINE PELEJO
}

\begin{abstract}
In this work, the authors consider a homogeneous system of linear equations of the form $A_{\alpha}^{\otimes N} \mathbf{x}=0$ arising from the distinguishability of two quantum operations by $N$ uses in parallel, where the coefficient matrix $A_{\alpha}$ depends on a real parameter $\alpha$. It was conjectured by Duan et al. that the system has a non-trivial nonnegative solution if and only if $\alpha$ lies in a certain interval $R_{N}$ depending on $N$. The authors affirm the necessity part of the conjecture and establish the sufficiency of the conjecture for $N \leq 10$ by presenting explicit non-trivial nonnegative solutions for the linear system.
\end{abstract}

Key words. Quantum channels, Parallel distinguishability.

AMS subject classifications. 46N60, 15A69.

1. Introduction. Let $M_{m, n}$ (respectively, $M_{n}$ ) be the set of $m \times n$ (respectively, $n \times n$ ) complex matrices. Denote by $H_{n}$ the set of $n \times n$ Hermitian matrices and by $D_{n}$ the set of $n \times n$ density matrices, which are positive semidefinite matrices with trace one.

In the mathematical framework of quantum mechanics, density matrices are used to describe the state of a quantum system. Quantum operations [5, 6] are trace-preserving, completely-positive linear maps from $M_{n}$ to $M_{m}$. It is known [2,4] that for a quantum operation $\mathcal{E}: M_{n} \longrightarrow M_{m}$, there exists a set of matrices $\left\{E_{1}, \ldots, E_{n_{0}}\right\} \subset M_{m, n}$, called a set of Choi-Kraus operators of $\mathcal{E}$, such that

$$
\sum_{j=1}^{n_{0}} E_{j}^{*} E_{j}=I_{n} \quad \text { and } \quad \mathcal{E}(X)=\sum_{j=1}^{n_{0}} E_{j} X E_{j}^{*} \quad \text { for any } X \in M_{n} .
$$

For example, the identity map on $M_{\ell}$, denoted by $\mathcal{I}_{\ell}$, has $\left\{I_{\ell}\right\}$ as Choi-Kraus operator.

Two quantum operations $\mathcal{E}: M_{n} \longrightarrow M_{m}$ and $\mathcal{F}: M_{n} \longrightarrow M_{m}$, with Choi-Kraus operators given by $\left\{E_{j}\right\}_{j=1}^{n_{0}}$ and $\left\{F_{k}\right\}_{k=1}^{n_{1}}$ are distinguishable by $N$ uses in parallel if for some integers $\ell, r$, there exists a nonzero vector $\mathbf{x} \in \mathbb{C}^{\ell^{r} \cdot n^{N}}$ such that

$$
Y_{1}=\left(\mathcal{I}_{\ell}^{\otimes r} \otimes \mathcal{E}^{\otimes N}\right)\left(\mathbf{x x}^{*}\right)=\sum_{j_{1}, \ldots, j_{N} \in\left\{1, \ldots, n_{0}\right\}}\left(I_{\ell}^{\otimes r} \otimes E_{j_{1}} \otimes \cdots \otimes E_{j_{N}}\right) \mathbf{x x}^{*}\left(I_{\ell}^{\otimes r} \otimes E_{j_{1}}^{*} \otimes \cdots \otimes E_{j_{N}}^{*}\right)
$$

*Received by the editors on September 2, 2019. Accepted for publication on February 28, 2020. Handling Editor: Shmuel Friedland. Corresponding Author: Chao Ma.

$\dagger$ Department of Mathematics, College of William and Mary, Williamsburg, Virginia, 23187, USA (ckli@math.wm.edu). Li is an affiliate member of the Institute for Quantum Computing, University of Waterloo; he is also an honorary professor of Shanghai University. His research was supported by USA NSF grant DMS 1331021, Simons Foundation grant 351047, and NNSF of China grants 11571220 and 11971294.

${ }^{\ddagger}$ College of Mathematics and Computer Science, Fuzhou University, Fuzhou, 350108, China (liuyue09@gmail.com). The research of Liu was supported by the National Natural Science Foundation of China grants 11571075 and 11871015.

$\S$ College of Arts and Sciences, Shanghai Maritime University, Shanghai 201306, China (machao0923@163.com). The research of Ma was supported by the National Natural Science Foundation of China grants 11601322 and 61573240.

IInstitute of Mathematics, College of Science, University of the Philippines Diliman, Quezon City 1101, Philippines (dcpelejo@math.upd.edu.ph). Pelejo was supported by PhD Incentive Award no. 191902 from the University of the Philippines Diliman Office of the Vice Chancellor for Research and Development. 
and

$$
Y_{2}=\left(\mathcal{I}_{\ell}^{\otimes r} \otimes \mathcal{F}^{\otimes N}\right)\left(\mathbf{x x}^{*}\right)=\sum_{k_{1}, \ldots, k_{N} \in\left\{1, \ldots, n_{1}\right\}}\left(I_{\ell}^{\otimes r} \otimes F_{k_{1}} \otimes \cdots \otimes F_{k_{N}}\right) \mathbf{x x}^{*}\left(I_{\ell}^{\otimes r} \otimes F_{k_{1}}^{*} \otimes \cdots \otimes F_{k_{N}}^{*}\right)
$$

are orthogonal, that is, $\operatorname{Tr}\left(Y_{1}^{*} Y_{2}\right)=0$. One may see [3] and its references for the background of the concept. In particular, the following results were obtained in [3, Theorems 1 and 2].

Proposition 1.1. Let $\mathcal{E}$ and $\mathcal{F}$ be two quantum operations with Choi-Kraus operators $\left\{E_{j}\right\}_{j=1}^{n_{0}}$ and $\left\{F_{k}\right\}_{k=1}^{n_{1}}$, respectively. Then $\mathcal{E}$ and $\mathcal{F}$ can be perfectly distinguished by $N$ uses in parallel if and only if there exists a density matrix $\rho \in\left(S_{\mathcal{E}, \mathcal{F}}^{\otimes N}\right)^{\perp}$, where

$$
S_{\mathcal{E}, \mathcal{F}}=\operatorname{Span}\left\{E_{j}^{*} F_{k} \mid 1 \leq j \leq n_{0}, 1 \leq k \leq n_{1}\right\} \quad \text { and } \quad S_{\mathcal{E}, \mathcal{F}}^{\otimes N}=\operatorname{Span}\left\{R^{\otimes N}: R \in S_{\mathcal{E}, \mathcal{F}}\right\} .
$$

Proposition 1.2. Any non-empty subset $T \subseteq M_{n}$ can be realized as a spanning set of $S_{\mathcal{E}, \mathcal{F}}$ of some pair of quantum operations $\mathcal{E}, \mathcal{F}$.

Here we give a short proof of Proposition 1.2:

Proof. Suppose Span $T$ has a basis $\left\{A_{1}, \ldots, A_{m}\right\} \subseteq M_{n}$. Consider the block diagonal matrix $\mathbf{A}=$ $A_{1} \oplus \cdots \oplus A_{m}$. If $\mathbf{A}$ has rank $\tilde{k}$, then $\mathbf{A}=\left[B_{1} \cdots B_{m}\right]^{*}\left[C_{1} \cdots C_{m}\right]$, where $B_{1}, \ldots, B_{m}, C_{1}, \ldots, C_{m}$ are $k \times n$ matrices with $k=\max \{\tilde{k}, n\}$. Let $M>0$ be such that $I_{n}-\frac{1}{M} \sum_{j=1}^{m} B_{j}^{*} B_{j}=B_{m+1}^{*} B_{m+1}$ and $I_{n}-\frac{1}{M} \sum_{j=1}^{m} C_{j}^{*} C_{j}=C_{m+1}^{*} C_{m+1}$ for some $k \times n$ matrices $B_{m+1}, C_{m+1}$. Let $E_{1}, \ldots, E_{m+1}, F_{1}, \ldots, F_{m+1} \in$ $M_{3 k, n}$ be such that

$$
\begin{gathered}
E_{j}^{*}=\frac{1}{\sqrt{M}}\left[B_{j}^{*} \mid 0_{n, 2 k}\right], \quad F_{j}^{*}=\frac{1}{\sqrt{M}}\left[C_{j}^{*} \mid 0_{n, 2 k}\right], \quad j=1, \ldots, m, \\
E_{m+1}^{*}=\left[0_{n, k}\left|B_{m+1}^{*}\right| 0_{n, k}\right], \quad F_{m+1}^{*}=\left[0_{n, 2 k} \mid C_{m+1}^{*}\right] .
\end{gathered}
$$

Then $\sum_{j=1}^{m+1} E_{j}^{*} E_{j}=\sum_{j=1}^{m+1} F_{j}^{*} F_{j}=I_{n}$, and

$$
\left[E_{1} \cdots E_{m+1}\right]^{*}\left[F_{1} \cdots F_{m+1}\right]=\frac{1}{M}\left(A_{1} \oplus \cdots \oplus A_{m} \oplus 0_{n}\right) .
$$

If the quantum channels from $M_{n}$ to $M_{3 k}$ have the sets of Choi-Kraus operators $\left\{E_{1}, \ldots, E_{m+1}\right\}$ and $\left\{F_{1}, \ldots, F_{m+1}\right\}$, then $\operatorname{Span}\left\{E_{i}^{*} F_{j}: 1 \leq i, j \leq m+1\right\}=\operatorname{Span}\left\{A_{1}, \ldots, A_{m}\right\}$.

In [3], the authors considered the quantum channels $\mathcal{E}$ and $\mathcal{F}$ with $S_{\mathcal{E}, \mathcal{F}}$ equal to the span of the set

$$
T_{\alpha}=\left\{\left[\begin{array}{ccc}
1 & 0 & 0 \\
0 & e^{i \alpha} & 0 \\
0 & 0 & 0
\end{array}\right],\left[\begin{array}{ccc}
0 & 0 & 0 \\
0 & 1 & 0 \\
0 & 0 & e^{i \alpha}
\end{array}\right]\right\}, \quad \alpha \in[0,2 \pi) .
$$

It is easy to see that the following conditions for a density operator $\rho=\left(\rho_{i j}\right) \in M_{3^{N}}$ are equivalent.

(a) The density operator $\rho \in\left(\operatorname{Span}\left(T_{\alpha}\right)^{\otimes N}\right)^{\perp}$.

(b) The diagonal density operator $\hat{\rho}=\operatorname{diag}\left(\rho_{11}, \ldots, \rho_{3^{N}, 3^{N}}\right) \in\left(\operatorname{Span}\left(T_{\alpha}\right)^{\otimes N}\right)^{\perp}$.

(c) The vector $\mathbf{x}=\left(\rho_{11}, \ldots, \rho_{3^{N}, 3^{N}}\right)^{t} \in \mathbb{C}^{3^{N}}$ satisfies the homogeneous equation

$$
A_{\alpha}^{\otimes N} \mathbf{x}=0 \quad \text { with } \quad A_{\alpha}=\left[\begin{array}{ccc}
1 & e^{i \alpha} & 0 \\
0 & 1 & e^{i \alpha}
\end{array}\right]
$$


By the above fact, one can focus on finding a non-trivial nonnegative vector $\mathbf{x} \in \mathbb{C}^{3^{N}}$ satisfying (1.1). Furthermore, the following remarks and conjecture were made in [3].

REMARK 1.3. If $\alpha \in\left[0, \frac{\pi}{2}\right)$, the space $\operatorname{Span}\left(T_{\alpha}\right)$ contains a positive operator. In this case $\left(\operatorname{Span}\left(T_{\alpha}\right)^{\otimes N}\right)^{\perp}$ does not contain a density matrix for any positive integer $N$. This makes the corresponding pair of quantum operations $\mathcal{E}, \mathcal{F}$, satisfying $S_{\mathcal{E}, \mathcal{F}}=\operatorname{Span}(T)$, indistinguishable. By taking the complex conjugate of equation (1.1), we see that there is a non-trivial nonnegative solution to $A_{\alpha}^{N} \mathbf{x}=0$ if and only if there is a non-trivial nonnegative solution to $A_{-\alpha}^{N} \mathbf{x}=A_{2 \pi-\alpha}^{N} \mathbf{x}=0$. Hence, we only need to focus on the case when $\alpha \in\left[\frac{\pi}{2}, \pi\right]$.

Conjecture 1.4. Let $\alpha \in\left[\frac{\pi}{2}, \pi\right]$. The equation (1.1) has a non-trivial nonnegative solution if and only if $\alpha \in\left[\frac{\pi}{2}+\frac{\pi}{2 N}, \pi\right]$.

In [3], the authors gave explicit solutions of the equation (1.1) for $N \leq 4$. Furthermore, in Section IV of the paper, it was shown that one may reduce the complexity of the equation (1.1) by finding solution with some symmetries imposed on its entries, and reduce the equation to another equation $C_{\alpha, N} \mathbf{y}=0$, where $C_{\alpha, N}$ is an $(N+1) \times \frac{(N+1)(N+2)}{2}$ matrix with full column rank. In Section 2, we will set up the system $C_{\alpha, N} \mathbf{y}=0$ and obtain another symmetry for the solution. In Section 3, we prove the necessity part of Conjecture 1.4, that is, if $\alpha \in\left[\frac{\pi}{2}, \pi\right]$ and equation (1.1) has a non-trivial nonnegative solution, then $\alpha \in\left[\frac{\pi}{2}+\frac{\pi}{2 N}, \pi\right]$. In Section 4, we present explicit non-trivial nonnegative solutions (1.1) for $\alpha \in\left[\frac{\pi}{2}+\frac{\pi}{2 N}, \pi\right]$ and $N \leq 10$. In Section 5, we provide some additional remarks that may help in studying the sufficiency part of the conjecture.

2. A reduction of the linear system. First, we label the entries of a vector $\mathbf{x} \in \mathbb{C}^{3^{N}}$ using ternary numbers. That is, we use the ternary number $\left(j_{0}, \ldots, j_{N-1}\right) \in\{0,1,2\}^{N}$, for the $j$-th entry of $\mathbf{x}$ when

$$
j=1+\sum_{p=0}^{N-1} j_{p} 3^{N-1-p} .
$$

For example, we will label the entries of $\mathbf{x} \in \mathbb{C}^{3^{2}}$ with $00,01,02,10,11,12,20,21,22$. In the same manner, we label the columns of $A_{\alpha}^{\otimes N}$ using ternary numbers. Meanwhile, we label the rows of $A_{\alpha}^{\otimes N}$ using binary numbers.

In $[3$, Section IV], it was shown that one may reduce the complexity of the equation (1.1) by finding solution $\mathbf{x}=\left[x_{J}\right]_{J \in\{0,1,2\}^{N}} \in \mathbb{C}^{3^{N}}$ with entries labeled by $J \in\{0,1,2\}^{N}$ such that $x_{J}=x_{\hat{J}}$ whenever $J=P \hat{J}$ for a permutation matrix $P \in M_{N}$, i.e., the ternary sequences $J$ and $\hat{J}$ have the same numbers of $0,1,2$ terms. We summarize the result in the following.

Proposition 2.1. If there is a non-trivial nonnegative solution $\mathbf{x}$ satisfying equation (1.1), then there is a non-trivial nonnegative solution $\hat{\mathbf{x}}=\left[\hat{x}_{J}\right]_{J \in\{0,1,2\}^{N}}$ such that

$$
\hat{x}_{j_{0}, \ldots, j_{N-1}}=\hat{x}_{k_{0}, \ldots, k_{N-1}}
$$

whenever there exists a permutation $\sigma \in S_{N}$ such that

$$
\left(j_{\sigma(0)}, \ldots, j_{\sigma(N-1)}\right)=\left(k_{0}, \ldots, k_{N-1}\right) .
$$

For a triple $\left(N_{0}, N_{1}, N_{2}\right)$ of nonnegative integers with $N_{0}+N_{1}+N_{2}=N$, define the set

$$
\left[N_{0}, N_{1}, N_{2}\right]=\left\{\left(j_{0}, j_{1}, \ldots, j_{N-1}\right) \in\{0,1,2\}^{N}: N_{\ell}=\#\left\{p: j_{p}=\ell\right\} \quad \text { for all } \ell \in\{0,1,2\}\right\},
$$


Electronic Journal of Linear Algebra, ISSN 1081-3810

A publication of the International Linear Algebra Society

Volume 36, pp. 198-209, April 2020.

of all ternary labels of length $N$ that contains $N_{0}$ digits equal to $0, N_{1}$ digits equal to 1 and $N_{2}$ digits equal to 2. For example, when $N=2$,

$$
[1,1,0]=\{01,10\}, \quad[0,1,1]=\{12,21\}, \quad[2,0,0]=\{00\} .
$$

Proposition 2.1 states that if there is a non-trivial nonnegative solution to $A_{\alpha}^{N} \mathbf{x}=0$, then there is a nontrivial nonnegative solution $\hat{\mathbf{x}}=\left[a_{J}\right]_{J \in\{0,1,2\}^{N}}$ such that $a_{J}=a_{K}$ whenever $J, K \in\left[N_{0}, N_{1}, N_{2}\right]$. Using this symmetry, $\hat{\mathbf{x}}$ has at most $p_{N}$ distinct entries, where $p_{N}$ is the number of nonnegative integer triples $\left(N_{0}, N_{1}, N_{2}\right)$ satisfying $N_{0}+N_{1}+N_{2}=N$. The total number of such triples equals the sum of solutions of $N_{0}+N_{1}=k$ for $k=0, \ldots, N$, and hence,

$$
p_{N}=1+\cdots+(N+1)=\frac{(N+1)(N+2)}{2} .
$$

For example, when $N=2$, we see from equation (2.3), that $\hat{x}$ has at most $p_{2}=6$ distinct entries. In fact, we may assume that the solution has the form:

$$
\hat{\mathbf{x}}^{T}=\left[\begin{array}{lllllllll}
x_{00} & x_{01} & x_{02} & x_{10} & x_{11} & x_{12} & x_{20} & x_{21} & x_{22}
\end{array}\right]=\left[\begin{array}{lllllllll}
a & b & c & b & d & e & c & e & f
\end{array}\right] .
$$

In the following, it is convenient to replace $A_{\alpha}$ by the matrix

$$
A_{\alpha}=\left[\begin{array}{ccc}
1 & 0 & -e^{2 i \alpha} \\
0 & 1 & e^{i \alpha}
\end{array}\right]=\left[\begin{array}{cc}
1 & -e^{i \alpha} \\
0 & 1
\end{array}\right]\left[\begin{array}{ccc}
1 & e^{i \alpha} & 0 \\
0 & 1 & e^{i \alpha}
\end{array}\right]=\left[\begin{array}{ccc}
1 & 0 & -z^{2} \\
0 & 1 & z
\end{array}\right] \quad \text { with } z=e^{i \alpha}
$$

Now, let us define a $3^{N} \times p_{N}$ matrix $Q_{N}$ by labeling its rows by ternary numbers in the usual order and labeling its first $N+1$ columns by $[N, 0,0],[N-1,0,1], \ldots,[1,0, N-1],[0,0, N]$, then its next $N$ columns by $[N-1,1,0],[N-2,1,1], \ldots,[0,1, N-1]$ and so on; then setting the $(i, j)$-th entry of $Q_{N}$ equal to 1 precisely when the ternary label of the $i$-th row is an element of the $j$-th column label as defined in equation (2.2). We can then define the following $2^{N} \times p_{N}$ matrix

$$
B_{\alpha, N}=A_{\alpha}^{\otimes N} Q_{N}
$$

Notice that for a non-trivial nonnegative solution $\hat{\mathbf{x}}$ satisfying the symmetry described in Proposition 2.1 , we have

$$
A_{\alpha}^{\otimes N} \hat{\mathbf{x}}=A_{\alpha}^{\otimes N} Q_{N} \mathbf{y}=B_{\alpha, N} \mathbf{y}
$$

for some nonzero nonnegative vector $\mathbf{y} \in \mathbb{C}^{p_{N}}$. Observe $B_{\alpha, N}$ for $N=2,3$ given below,

$$
\begin{aligned}
& {[200] \quad[101] \quad[002] \quad[110] \quad[011] \quad[020]} \\
& B_{\alpha, 2}=\begin{array}{c}
{[00]} \\
{[01]} \\
{[10]} \\
{[11]}
\end{array}\left[\begin{array}{cccccc}
1 & -2 z^{2} & z^{4} & 0 & 0 & 0 \\
0 & z & -z^{3} & 1 & -z^{2} & 0 \\
0 & z & -z^{3} & 1 & -z^{2} & 0 \\
0 & 0 & z^{2} & 0 & 2 z & 1
\end{array}\right]
\end{aligned}
$$

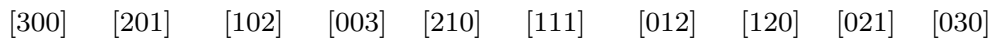

$$
\begin{aligned}
& \begin{aligned}
{[000] } \\
{[001] } \\
{[010] }
\end{aligned}\left[\begin{array} { c c c c c c c c c c c } 
{ 1 } & { - 3 z ^ { 2 } } & { 3 z ^ { 4 } } & { - z ^ { 6 } } & { 0 } & { 0 } & { 0 } & { 0 } & { 0 } & { 0 } \\
{ 0 } & { z } & { - 2 z ^ { 3 } } & { z ^ { 5 } } & { 1 } & { - 2 z ^ { 2 } } & { z ^ { 4 } } & { 0 } & { 0 } & { 0 } \\
{ 0 } & { z } & { - 2 z ^ { 3 } } & { z ^ { 5 } } & { 1 } & { - 2 z ^ { 2 } } & { z ^ { 4 } } & { 0 } & { 0 } & { 0 } \\
{ } & { [ 0 1 1 ] } \\
{ } & { [ 1 0 0 ] } \\
{ } & { [ 1 0 1 ] } \\
{ } & { [ 1 1 0 ] } \\
{ } & { [ 1 1 1 ] }
\end{array} \left[\begin{array}{ccccccc}
B^{4}, 3 \\
0
\end{array}\right.\right.
\end{aligned}
$$


Proposition 2.2. Let $B_{\alpha, N}$ be defined as in equation (2.5). Then:

(a) $\operatorname{rank}\left(B_{\alpha, N}\right)=N+1$.

(b) The first $N+1$ columns of $B_{\alpha, N}$ are linearly independent.

(c) If the digits of the binary labels of rows $J$ and $K$ have the same number of zeros (equivalently, the same number of ones), then the $J$-th and $K$-th rows of $B_{\alpha, N}$ are identical.

(d) Let $J=00 \cdots \underbrace{11 \ldots 1}_{j}$. Then

$$
\left(B_{\alpha, N}\right)_{J ;\left[N_{0}, N_{1}, N_{2}\right]}=\left(\begin{array}{c}
N-j \\
N_{0}
\end{array}\right) \cdot\left(-z^{2}\right)^{N-j-N_{0}} \cdot\left(\begin{array}{c}
j \\
N_{1}
\end{array}\right) z^{j-N_{1}},
$$

where we agree that $\left(\begin{array}{l}n \\ k\end{array}\right)=0$ whenever $k>n$.

Proof. Let $A_{\alpha}$ be defined as in equation (2.4). Denote its entries by $a_{j, \ell}$ where $j \in\{0,1\}$ and $\ell \in\{0,1,2\}$. One can check that if $J=\left(j_{1}, \ldots, j_{N}\right) \in\{0,1\}^{N}$ and $L=\left(\ell_{1}, \ldots, \ell_{N}\right) \in\{0,1,2\}^{N}$, then the $(J, L)$-th entry of $A_{\alpha}^{\otimes N}$ is $\prod_{s=1}^{N} a_{j_{s}, \ell_{s}}$.

We first prove (c). Since $J$ and $K$ have the same number of zeros, there exists $\sigma \in S_{N}$ such that $J=\sigma(K)$.

Let $N_{0}, N_{1}, N_{2}$ be nonnegative integers with $N_{0}+N_{1}+N_{2}=N, \tau \in S_{N}$, write

$$
\tau\left(\left[N_{0}, N_{1}, N_{2}\right]\right):=\left\{\tau(L) \mid L \in\left[N_{0}, N_{1}, N_{2}\right]\right\}
$$

It is easy to verify that $\tau\left(\left[N_{0}, N_{1}, N_{2}\right]\right)=\left[N_{0}, N_{1}, N_{2}\right]$ for any $\tau$. Then

$$
\begin{aligned}
\left(B_{\alpha, N}\right)_{J ;\left[N_{0}, N_{1}, N_{2}\right]} & \sum_{L=\ell_{1} \ell_{2} \cdots \ell_{N} \in\left[N_{0}, N_{1}, N_{2}\right]}\left(A_{\alpha}^{\otimes N}\right)_{J, L} \\
& =\sum_{L=\ell_{1} \ell_{2} \cdots \ell_{N} \in\left[N_{0}, N_{1}, N_{2}\right]} \prod_{s=1}^{N}\left(A_{\alpha}\right)_{j_{s}, \ell_{s}} \\
& =\sum_{\sigma(L) \in\left[N_{0}, N_{1}, N_{2}\right]} \prod_{s=1}^{N}\left(A_{\alpha}\right)_{j_{\sigma(s)}, \ell_{\sigma(s)}} \\
& =\sum_{L \in\left[N_{0}, N_{1}, N_{2}\right]} \prod_{s=1}^{N}\left(A_{\alpha}\right)_{k_{s}, \ell_{s}} \\
= & \left(B_{\alpha, N}\right)_{K ;\left[N_{0}, N_{1}, N_{2}\right]}
\end{aligned}
$$

where $J=j_{1} j_{2} \cdots j_{N}$ and $K=k_{1} k_{2} \cdots k_{N}$. Thus, the $J$-th and $K$-th rows of $B_{\alpha, N}$ are identical.

Note that

$$
\left(B_{\alpha, N}\right)_{J ;\left[N_{0}, N_{1}, N_{2}\right]}=\sum_{L=\ell_{1} \ell_{2} \cdots \ell_{N} \in\left[N_{0}, N_{1}, N_{2}\right]} \prod_{s=1}^{N-j} a_{0, \ell_{s}} \prod_{t=N-j+1}^{N} a_{1, \ell_{t}}
$$

Let $L \in\left[N_{0}, N_{1}, N_{2}\right]$ corresponding to a nonzero term in the formula (2.7). Since $a_{10}=0$, then $\left\{s \mid \ell_{s}=0\right\} \subseteq$ $[N-j]$. Since \# $\left.\# s \mid \ell_{s}=0\right\}=N_{0}$, there are $\left(\begin{array}{c}N-j \\ N_{0}\end{array}\right)$ different choices for the positions of 0s in $L$. Now suppose that the positions of 0 s have been chosen, then for $s \in[N-j] \backslash\left\{s \mid \ell_{s}=0\right\}, \ell_{s}$ can't be 1 since $a_{01}=0$. Thus, there are $N-j-N_{0}$ terms of $-z^{2}$ in the first product. 
For the second product, $\ell_{N-j+1} \cdots \ell_{N}$ must contain all the $N_{1}$ 1s in $L$ since $a_{01}=0$, and there are $\left(\begin{array}{c}j \\ N_{1}\end{array}\right)$ different choices. After the positions of $1 \mathrm{~s}$ been chosen, all the $\ell_{t}$ left must be 2 , and thus, the corresponding terms in the product are $z$, yielding that the formula (2.6) holds.

By formula (2.6), the submatrix of $B_{\alpha, N}$ obtained by taking only the rows labeled by

$$
00 \cdots 00,00 \cdots 01, \ldots, 11 \cdots 11
$$

and columns labeled by

$$
[N, 0,0],[N-1,0,1], \ldots,[1,0, N-1],[0,0, N]
$$

is an upper triangular matrix with nonzero diagonals. Thus, (a) and (b) hold.

Now, define the $(N+1) \times p_{N}$ matrix $C_{\alpha, N}$ as the submatrix of $B_{\alpha, N}$ obtained by taking only the rows labeled by $00 \cdots 00,00 \cdots 01, \ldots, 11 \cdots 11$. This makes $C_{\alpha, N}$ a full row rank matrix such that $B_{\alpha, N} \mathbf{y}=0$ if and only if $C_{\alpha, N} \mathbf{y}=0$. We illustrate $C_{\alpha, 2}$ and $C_{\alpha, 3}$ below,

$$
\begin{aligned}
& {[200] \quad[101] \quad[002] \quad[110] \quad[011] \quad[020]} \\
& C_{\alpha, 2}=\left[\begin{array}{l}
{[00]} \\
{[01]}
\end{array}\left[\begin{array}{cccccc}
1 & -2 z^{2} & z^{4} & 0 & 0 & 0 \\
0 & z & -z^{3} & 1 & -z^{2} & 0 \\
0 & 0 & z^{2} & 0 & 2 z & 1
\end{array}\right]\right.
\end{aligned}
$$

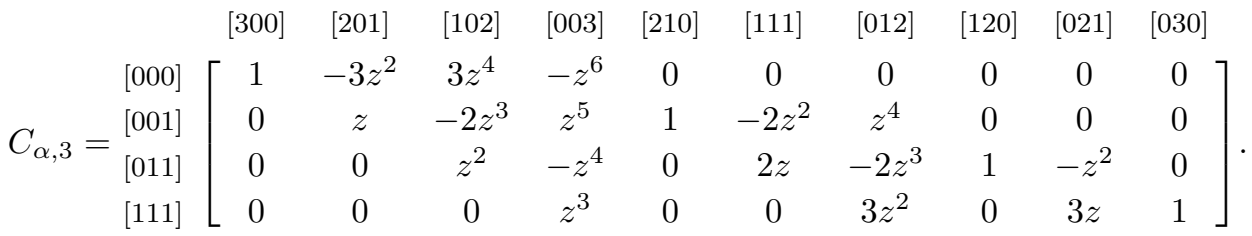

Notice that we can write $C_{\alpha, N}$ as

$$
C_{\alpha, N}=\left[\begin{array}{c|c|c|c|c}
\Gamma_{N} & 0_{1, N} & 0_{2, N-1} & \ldots & 0_{N, 1} \\
& D_{N, 1} \Gamma_{N-1} & D_{N, 2} \Gamma_{N-2} & & D_{N, N} \Gamma_{0}
\end{array}\right],
$$

where for $n=N, \ldots, 0$, and $k \leq n, D_{n, k}$ is the diagonal matrix $\operatorname{diag}\left(\left(\begin{array}{c}k \\ k\end{array}\right),\left(\begin{array}{c}k+1 \\ k\end{array}\right), \ldots,\left(\begin{array}{l}n \\ k\end{array}\right)\right) \in M_{n-k+1}$, and

$$
\Gamma_{n}=\left[\begin{array}{cccc}
1 & & & \\
& z & & \\
& \ddots & \\
& & \ddots & \\
& & & z^{n}
\end{array}\right]\left[\begin{array}{ccccc}
1 & \left(\begin{array}{c}
n \\
1
\end{array}\right)\left(-z^{2}\right) & \left(\begin{array}{c}
n \\
2
\end{array}\right)\left(-z^{2}\right)^{2} & \ldots & \left(\begin{array}{c}
n \\
n
\end{array}\right)\left(-z^{2}\right)^{n} \\
0 & 1 & \left(\begin{array}{c}
n-1 \\
1
\end{array}\right)\left(-z^{2}\right) & \cdots & \left(\begin{array}{c}
n-1 \\
n-1
\end{array}\right)\left(-z^{2}\right)^{n-1} \\
\vdots & \vdots & \vdots & & \vdots \\
0 & 0 & 0 & \ldots & 1
\end{array}\right]
$$

So, $\Gamma_{n}$ is an $(n+1) \times(n+1)$ upper triangular matrix whose $(j, k)$-th entry is given by

$$
\begin{cases}(-1)^{k-j}\left(\begin{array}{c}
n+1-j \\
k-j
\end{array}\right) z^{j-1+2(k-j)} & \text { if } k \geq j \\
0 & \text { if } j>k .\end{cases}
$$


3. Necessity of Conjecture 1.4. To prove the necessity of Conjecture 1.4, we demonstrate another symmetry one may impose on the solution of $\mathbf{x}$ of the equation (1.1).

Proposition 3.1. Suppose $\mathbf{x} \in \mathbb{R}^{3^{N}}$ satisfies equation (1.1), and $\hat{\mathbf{x}}$ is obtained from $\mathbf{x}$ by exchanging the entries $\mathbf{x}_{j}=\mathbf{x}_{3^{N}-j}$ whenever $1 \leq j \leq\left(3^{N}-1\right) / 2$. Then $A^{\otimes N} \hat{\mathbf{x}}=0$.

Note that if the entries of $\mathbf{x}$ and $\hat{\mathbf{x}}$ are labeled by $x_{j_{1} \cdots j_{N}}$ and $\hat{x}_{j_{1} \cdots j_{N}}$ using ternary sequences $j_{1} \cdots j_{N} \in$ $\{0,1,2\}^{N}$, then $x_{j_{1} \cdots j_{N}}=\hat{x}_{\left(2-j_{1}\right) \cdots\left(2-j_{N}\right)}$.

Proof. Let $\tilde{A}_{\alpha}=\left[\begin{array}{ccc}1 & e^{i \alpha} & 0 \\ 0 & e^{-i \alpha} & 1\end{array}\right], J=\left[\begin{array}{ll}0 & 1 \\ 1 & 0\end{array}\right]$ and $K=\left[\begin{array}{lll}0 & 0 & 1 \\ 0 & 1 & 0 \\ 1 & 0 & 0\end{array}\right]$. Then for $A_{\alpha}$ defined in (1.1),

$$
\left[\begin{array}{cc}
1 & 0 \\
0 & e^{-i \alpha}
\end{array}\right] A_{\alpha}=\tilde{A}_{\alpha}=J \overline{\tilde{A}_{\alpha}} K
$$

Thus, $\mathbf{x} \in \operatorname{Null}\left(A_{\alpha}^{\otimes N}\right)$ if and only if $\mathbf{x} \in \operatorname{Null}\left(\tilde{A}_{\alpha}^{\otimes N}\right)$. Additionally, if $\mathbf{x}$ is real,

$$
\mathbf{x} \in \operatorname{Null}\left(\tilde{A}_{\alpha}^{\otimes N}\right) \quad \Longleftrightarrow \quad \tilde{A}_{\alpha}^{\otimes N} \mathbf{x}=\mathbf{0} \quad \Longleftrightarrow \quad \tilde{A}_{\alpha}^{\otimes N} \mathbf{x}=\mathbf{0} \quad \Longleftrightarrow \quad K^{\otimes N} \mathbf{x} \in \operatorname{Null}\left(\tilde{A}_{\alpha}^{\otimes N}\right) .
$$

So, $\hat{x}_{j_{1} \cdots j_{N}}=\hat{x}_{2-j_{1} \cdots 2-j_{N}}$. Thus, we can assume that $x_{i_{1} i_{2} \cdots i_{n}}=x_{\left(2-i_{1}\right)\left(2-i_{2}\right) \cdots\left(2-i_{N}\right)}$.

By the above proposition and the discussion in Section 2, we see that the system $A_{\alpha}^{\otimes N} \mathbf{x}=0$ has a non-trivial nonnegative solution if and only if the system $C_{\alpha, N} \mathbf{y}=0$ has a non-trivial nonnegative solution y. We have the following.

THEOREM 3.2. Let $\alpha \in\left[\frac{\pi}{2}, \pi\right]$. If the equation $C_{\alpha, N} \mathbf{y}=0$ has a non-trivial nonnegative solution $\mathbf{y}$, then $\alpha \in\left[\frac{\pi}{2}+\frac{\pi}{2 N}, \pi\right]$.

Proof. We consider the reduced equation $C_{\alpha, N} \mathbf{y}=0$ with $z=e^{i \alpha}$ as shown in Section 2. Let

$$
\begin{aligned}
\mathbf{y} & =\left(y_{[N 00]}, y_{[(N-1) 01]}, \ldots, y_{[00 N]}, y_{[(N-1) 10]}, \ldots, y_{[01(N-1)]}, \ldots, \ldots, y_{[0 N 0]}\right)^{t} \\
& =\left(y_{0,0}, y_{0,1}, \ldots, y_{0, N}, y_{1,0}, \ldots, y_{1, N-1}, \ldots, y_{N, 0}\right)^{t}
\end{aligned}
$$

be a nonnegative solution of $C_{\alpha, N} \mathbf{y}=0$. We will show that if $\alpha \in\left[\frac{\pi}{2}, \frac{\pi}{2}+\frac{\pi}{2 N}\right)$, then $\mathbf{y}$ is a zero vector, which is a contradiction.

Case 1. $N$ is even. Since $y_{j, k}=y_{j, N-j-k}$, we may rewrite the first equation of the linear system as

$$
\sum_{k=0}^{N / 2-1}(-1)^{k}\left(\begin{array}{c}
N \\
k
\end{array}\right)\left(z^{2 k}+z^{2 N-2 k}\right) y_{0, k}+(-1)^{N / 2}\left(\begin{array}{c}
N \\
N / 2
\end{array}\right) z^{N} y_{0, N / 2}=0
$$

Divided by $z^{N},(3.8)$ reduces to

$$
\sum_{k=0}^{N / 2-1}(-1)^{k}\left(\begin{array}{c}
N \\
k
\end{array}\right) 2 \cos ((N-2 k) \alpha) y_{0, k}+(-1)^{N / 2}\left(\begin{array}{c}
N \\
N / 2
\end{array}\right) y_{0, N / 2}=0
$$

Let $\theta=\alpha-\frac{\pi}{2}$. Since we assume that $\alpha \in\left[\frac{\pi}{2}, \frac{\pi}{2}+\frac{\pi}{2 N}\right)$, then $\theta \in\left[0, \frac{\pi}{2 N}\right)$ so that $\cos (m \theta)$ are all positive for $1 \leq m \leq N$. 
Now replace $\alpha$ in (3.9) with $\theta$, we have

$$
(-1)^{N / 2}\left(\sum_{k=0}^{N / 2-1}\left(\begin{array}{c}
N \\
k
\end{array}\right) 2 \cos ((N-2 k) \theta) y_{0, k}+\left(\begin{array}{c}
N \\
N / 2
\end{array}\right) y_{0, N / 2}\right)=0 .
$$

Since all the coefficients of $y_{0, k}$ are nonnegative, $y_{0, k}=0$ for all $k=0,1, \ldots, N$.

Case 2. $N$ is odd. Since $y_{j, k}=y_{j, N-j-k}$, we may rewrite the first equation of the linear system as

$$
\sum_{k=0}^{(N-1) / 2}(-1)^{k}\left(\begin{array}{l}
N \\
k
\end{array}\right)\left(z^{2 k}-z^{2 N-2 k}\right) y_{0, k}=0
$$

Dividing the equation by $i z^{N}$, and replacing $\alpha$ with $\theta=\alpha-\frac{\pi}{2}$, we get

$$
(-1)^{\frac{N+1}{2}}\left(\sum_{k=0}^{(N-1) / 2}\left(\begin{array}{l}
N \\
k
\end{array}\right) 2 \cos ((N-2 k) \theta) y_{0, k}\right)=0,
$$

by the same reason as the even case, $y_{0, k}$ needs to be 0 for all $k=0,1, \ldots, N$.

For $y_{1,0}, y_{1,1}, \ldots, y_{1, N-1}$, since it is already proved that when $\alpha \in\left[\frac{\pi}{2}, \frac{\pi}{2}+\frac{\pi}{2 N}\right), y_{0,0}=y_{0,1}=\cdots=$ $y_{0, N}=0$, the second equation of $C_{\alpha, N} \mathbf{y}=0$ becomes the same as the first equation of $C_{\alpha, N-1} \mathbf{y}=0$. By induction on $N$, since $\left[\frac{\pi}{2}, \frac{\pi}{2}+\frac{\pi}{2 N}\right) \subseteq\left[\frac{\pi}{2}, \frac{\pi}{2}+\frac{\pi}{2(N-1)}\right)$, we have $y_{1,0}=y_{1,1}=\cdots=y_{1, N-1}=0$. Furthermore, by induction on $j$, we have $y_{j, 0}=\cdots=y_{j, N-j}=0$ for $j=0,1, \ldots, N$, which means $\mathbf{y}=0$, completing the proof.

Corollary 3.3. Let $\alpha \in\left[\frac{\pi}{2}, \pi\right]$. If the equation $A_{\alpha}^{\otimes N} \mathbf{x}=0$ has a non-trivial nonnegative solution $\mathbf{x}$, then $\alpha \in\left[\frac{\pi}{2}+\frac{\pi}{2 N}, \pi\right]$.

4. Explicit solution of the system $C_{\alpha, N} \mathbf{y}=0$ when $N \leq 10$. Note that if $\alpha \in\left[\frac{\pi}{2}+\frac{\pi}{2 N}, \pi\right] \subseteq$ $\left[\frac{\pi}{2}+\frac{\pi}{2(N+1)}, \pi\right]$ and $\mathbf{x} \in \mathbb{C}^{3^{N}}$ satisfy $A_{\alpha}^{\otimes N} \mathbf{x}=0$, then for any nonnegative vector $\mathbf{y} \in \mathbb{C}^{3}$, we have $A_{\alpha}^{\otimes(N+1)}(\mathbf{x} \otimes$ $\mathbf{y})=0$. Thus, for $N \leq 10$, it is enough to find a non-trivial nonnegative solution to $C_{\alpha, N} \mathbf{y}=0$ when $\alpha \in\left[\frac{\pi}{2}+\frac{\pi}{2 N}, \frac{\pi}{2}+\frac{\pi}{2(N-1)}\right)$. In the next lemma, we determine the exact location of $e^{i k \alpha}$ in the Argand plane. Let $Q_{1}, Q_{2}, Q_{3}, Q_{4}$ denote the four quadrants of the complex plane.

LemMa 4.1. For $N \geq 2$, let

$$
\theta \in\left[\frac{\pi}{2 N}, \frac{\pi}{2(N-1)}\right) \quad \text { and } \quad \alpha=\theta+\frac{\pi}{2} .
$$

Then $N \alpha \in Q_{N+2}(\bmod 4)$, and for $k<N$ we have $k \alpha \in Q_{k+1}(\bmod 4)$.

Proof. Note that $[a, b] \subseteq Q_{r}$ if and only if there exists $\ell$ such that $r \equiv \ell+1(\bmod 4)$ and $\frac{\ell}{2} \pi \leq a \leq$ $b \leq \frac{\ell+1}{2} \pi$. Since $\alpha \in\left[\pi\left(\frac{1}{2}+\frac{1}{2 N}\right), \pi\left(\frac{1}{2}+\frac{1}{2(N-1)}\right)\right)$, then $N \alpha \in\left[\pi\left(\frac{N+1}{2}\right), \pi\left(\frac{N+1}{2}+\frac{1}{2(N-1)}\right)\right)$. Note that $\frac{1}{2(N-1)} \leq \frac{1}{2}$, and hence, if $\ell=N+1$, then $\alpha \in Q_{r}$ where $r \equiv \ell+1 \equiv N+2(\bmod 4)$. On the other hand, if $0 \leq k<N$, then $\frac{k}{2(N-1)} \leq \frac{1}{2}$. Note that $k \alpha \in\left[\pi\left(\frac{k}{2}+\frac{k}{2 N}\right), \pi\left(\frac{k}{2}+\frac{k}{2(N-1)}\right)\right)$. Thus, if $\ell=k$ then $k \alpha \in Q_{r}$ where $r \equiv \ell+1 \equiv k+1(\bmod 4)$.

We now present some explicit non-trivial nonnegative solutions to $C_{\alpha, N} \mathbf{y}=0$. One can use the preceding lemma to verify that the given $\mathbf{y}$ is nonzero and nonnegative. 
1. For $N=1$, we have $\alpha=\pi$ and a non-trivial nonnegative solution given by

$$
\mathbf{y}^{T}=\left[\begin{array}{ccc}
{[100]} & {[001]} & {[010]} \\
1 & 1 & 1
\end{array}\right]
$$

2. For $N=2$, we have $\alpha \in\left[\frac{3 \pi}{4}, \pi\right)$ and a non-trivial nonnegative solution given by

$$
\mathbf{y}^{T}=\left[\begin{array}{cccccc}
{[200]} & {[101]} & {[002]} & {[110]} & {[011]} & {[020]} \\
1 & \cos 2 \alpha & 1 & -\cos \alpha & -\cos \alpha & 1
\end{array}\right] .
$$

3. For $N=3$, we have $\alpha \in\left[\frac{2 \pi}{3}, \frac{3 \pi}{4}\right)$ and a non-trivial nonnegative solution given by

$$
\mathbf{y}^{T}=\left[\begin{array}{cccccccccc}
{[300]} & {[201]} & {[102]} & {[003]} & {[210]} & {[111]} & {[012]} & {[120]} & {[021]} & {[030]} \\
3 \sin \alpha & \sin 3 \alpha & \sin 3 \alpha & 3 \sin \alpha & -\sin 2 \alpha & -\sin 2 \alpha & -\sin 2 \alpha & \sin \alpha & \sin \alpha & 0
\end{array}\right] .
$$

4. For $N=4$, we have $\alpha \in\left[\frac{5 \pi}{8}, \frac{2 \pi}{3}\right)$ and a non-trivial nonnegative solution given by

$$
\begin{aligned}
& \mathbf{y}^{T}=\left[\begin{array}{lllll}
\mathbf{a}_{0} & \mathbf{a}_{1} & \mathbf{a}_{2} & \mathbf{a}_{3} & \mathbf{a}_{4}
\end{array}\right] \text {, where } \\
& {[400] \quad[301] \quad[202] \quad[103] \quad[004]} \\
& \mathbf{a}_{0}=\left[\begin{array}{lllll}
6 & 0 & -2 \cos 4 \alpha & 0 & 6
\end{array}\right], \\
& {[310] \quad[211] \quad[112] \quad[013]} \\
& \mathbf{a}_{1}=\left[\begin{array}{llll}
-3 \cos \alpha & \cos 3 \alpha & \cos 3 \alpha & -3 \cos \alpha
\end{array}\right], \\
& {\left[\begin{array}{llllll}
{[220]} & {[121]} & {[022]} & {[130]} & {[031]} & {[040]}
\end{array}\right.} \\
& {\left[\begin{array}{lll}
\mathbf{a}_{2} & \mathbf{a}_{3} & \mathbf{a}_{4}
\end{array}\right]=\left[\begin{array}{llllll}
2 & 0 & 2 & -3 \cos \alpha & -3 \cos \alpha & 6
\end{array}\right] .}
\end{aligned}
$$

5. For $N=5$, we have $\alpha \in\left[\frac{3 \pi}{5}, \frac{5 \pi}{8}\right)$ and a non-trivial nonnegative solution given by

$$
\left.\begin{array}{rl}
\mathbf{y}^{T}=\left[\begin{array}{llllll}
\mathbf{a}_{0} & \mathbf{a}_{1} & \mathbf{a}_{2} & \mathbf{a}_{3} & \mathbf{a}_{4} & \mathbf{a}_{5}
\end{array}\right], \text { where } \\
\mathbf{a}_{0}= & \left.\begin{array}{ccccccc}
{[500]} & {[401]} & {[302]} & {[203]} & {[104]} & {[005]} \\
20 \sin \alpha & 0 & -2 \sin 5 \alpha & -2 \sin 5 \alpha & 0 & 20 \sin \alpha
\end{array}\right], \\
\mathbf{a}_{1}= & {\left[\begin{array}{ccccccc}
-4 \sin 2 \alpha & \sin 4 \alpha & 2 \sin 4 \alpha & \sin 4 \alpha & -4 \sin 2 \alpha
\end{array}\right],} \\
\mathbf{a}_{2}= & {\left[\begin{array}{lllllll}
3 \sin \alpha & -\sin 3 \alpha & -\sin 3 \alpha & 3 \sin \alpha
\end{array}\right],}
\end{array}\right]
$$

6. For $N=6$, we have $\alpha \in\left[\frac{7 \pi}{12}, \frac{3 \pi}{5}\right)$ and a non-trivial nonnegative solution given by

$$
\begin{aligned}
& \mathbf{y}^{T}=\left[\begin{array}{lllllll}
\mathbf{a}_{0} & \mathbf{a}_{1} & \mathbf{a}_{2} & \mathbf{a}_{3} & \mathbf{a}_{4} & \mathbf{a}_{5} & \mathbf{a}_{6}
\end{array}\right] \text {, where } \\
& \mathbf{a}_{0}=\left[\begin{array}{lllllll}
20 & 0 & 0 & 2 \cos 6 \alpha & 0 & 0 & 20
\end{array}\right] \text {, } \\
& \mathbf{a}_{1}=\left[\begin{array}{llllll}
-10 \cos \alpha & 0 & -\cos 5 \alpha & -\cos 5 \alpha & 0 & -10 \cos \alpha
\end{array}\right], \\
& {\left[\begin{array}{ll}
\mathbf{a}_{2} & \mathbf{a}_{3}
\end{array}\right]=\left[\begin{array}{lllllllll}
3 & 0 & \cos 4 \alpha & 0 & 3 & \cos 3 \alpha & 0 & 0 & \cos 3 \alpha
\end{array}\right],} \\
& {\left[\begin{array}{lll}
\mathbf{a}_{4} & \mathbf{a}_{5} & \mathbf{a}_{6}
\end{array}\right]=\left[\begin{array}{llllll}
-2 \cos 2 \alpha & 0 & -2 \cos 2 \alpha & 0 & 0 & 5
\end{array}\right] \text {. }}
\end{aligned}
$$


Electronic Journal of Linear Algebra, ISSN 1081-3810

7. For $N=7$, we have $\alpha \in\left[\frac{4 \pi}{7}, \frac{7 \pi}{12}\right)$ and a non-trivial nonnegative solution given by

$$
\begin{aligned}
& \mathbf{y}^{T}=\left[\begin{array}{llllllll}
\mathbf{a}_{0} & \mathbf{a}_{1} & \mathbf{a}_{2} & \mathbf{a}_{3} & \mathbf{a}_{4} & \mathbf{a}_{5} & \mathbf{a}_{6} & \mathbf{a}_{7}
\end{array}\right] \text {, where } \\
& \mathbf{a}_{0}=\left[\begin{array}{llllllll}
140 \sin \alpha & 0 & 0 & 4 \sin 7 \alpha & 4 \sin 7 \alpha & 0 & 0 & 140 \sin \alpha
\end{array}\right], \\
& \mathbf{a}_{1}=\left[\begin{array}{lllllll}
-30 \sin 2 \alpha & 0 & -2 \sin 6 \alpha & -4 \sin 6 \alpha & -2 \sin 6 \alpha & 0 & -30 \sin 2 \alpha
\end{array}\right], \\
& \mathbf{a}_{2}=\left[\begin{array}{llllll}
20 \sin \alpha & 0 & 2 \sin 5 \alpha & 2 \sin 5 \alpha & 0 & 20 \sin \alpha
\end{array}\right] \\
& \mathbf{a}_{3}=\left[\begin{array}{lllll}
2 \sin 4 \alpha-4 \sin 2 \alpha & \sin 4 \alpha & 0 & \sin 4 \alpha & 2 \sin 4 \alpha-4 \sin 2 \alpha
\end{array}\right], \\
& \mathbf{a}_{4}=\left[\begin{array}{llll}
-4 \sin 3 \alpha+6 \sin \alpha & -2 \sin 3 \alpha & -2 \sin 3 \alpha & -4 \sin 3 \alpha+6 \sin \alpha
\end{array}\right], \\
& {\left[\begin{array}{lll}
\mathbf{a}_{5} & \mathbf{a}_{6} & \mathbf{a}_{7}
\end{array}\right]=\left[\begin{array}{llllll}
0 & 0 & 0 & 10 \sin \alpha & 10 \sin \alpha & 0
\end{array}\right] .}
\end{aligned}
$$

8. For $N=8$, we have $\alpha \in\left[\frac{9 \pi}{16}, \frac{4 \pi}{7}\right)$ and a non-trivial nonnegative solution given by

$$
\begin{aligned}
& \mathbf{y}^{T}=\left[\begin{array}{lllllllll}
\mathbf{a}_{0} & \mathbf{a}_{1} & \mathbf{a}_{2} & \mathbf{a}_{3} & \mathbf{a}_{4} & \mathbf{a}_{5} & \mathbf{a}_{6} & \mathbf{a}_{7} & \mathbf{a}_{8}
\end{array}\right] \text {, where } \\
& \mathbf{a}_{0}=\left[\begin{array}{lllllllll}
140 & 0 & 0 & 0 & -4 \cos 8 \alpha & 0 & 0 & 0 & 140
\end{array}\right], \\
& \mathbf{a}_{1}=\left[\begin{array}{llllllll}
-70 \cos \alpha & 0 & 0 & 2 \cos 7 \alpha & 2 \cos 7 \alpha & 0 & 0 & -70 \cos \alpha
\end{array}\right], \\
& \mathbf{a}_{2}=\left[\begin{array}{lllllll}
20 & 0 & 0 & -2 \cos 6 \alpha & 0 & 0 & 20
\end{array}\right] \text {, } \\
& \mathbf{a}_{3}=\left[\begin{array}{llllll}
5 \cos 3 \alpha & -\cos 5 \alpha & 0 & 0 & -\cos 5 \alpha & 5 \cos 3 \alpha
\end{array}\right], \\
& \mathbf{a}_{4}=\left[\begin{array}{lllll}
-8 \cos 2 \alpha & 2 \cos 4 \alpha & 0 & 2 \cos 4 \alpha & -8 \cos 2 \alpha
\end{array}\right],
\end{aligned}
$$

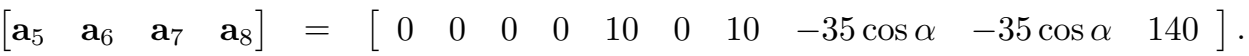

9. For $N=9$, we have $\alpha \in\left[\frac{5 \pi}{9}, \frac{9 \pi}{16}\right)$ and a non-trivial nonnegative solution given by

$$
\begin{aligned}
& \mathbf{y}^{T}=\left[\begin{array}{llllllllll}
\mathbf{a}_{0} & \mathbf{a}_{1} & \mathbf{a}_{2} & \mathbf{a}_{3} & \mathbf{a}_{4} & \mathbf{a}_{5} & \mathbf{a}_{6} & \mathbf{a}_{7} & \mathbf{a}_{8} & \mathbf{a}_{9}
\end{array}\right] \text {, where } \\
& \mathbf{a}_{0}=\left[\begin{array}{llllllllll}
504 \sin \alpha & 0 & 0 & 0 & -4 \sin 9 \alpha & -4 \sin 9 \alpha & 0 & 0 & 0 & 504 \sin \alpha
\end{array}\right], \\
& \mathbf{a}_{1}=\left[\begin{array}{lllllllll}
-112 \sin 2 \alpha & 0 & 0 & 2 \sin 8 \alpha & 4 \sin 8 \alpha & 2 \sin 8 \alpha & 0 & 0 & -112 \sin 2 \alpha
\end{array}\right], \\
& \mathbf{a}_{2}=\left[\begin{array}{llllllll}
70 \sin \alpha & 0 & 0 & -2 \sin 7 \alpha & -2 \sin 7 \alpha & 0 & 0 & 70 \sin \alpha
\end{array}\right],
\end{aligned}
$$

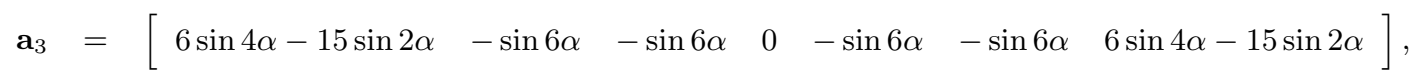

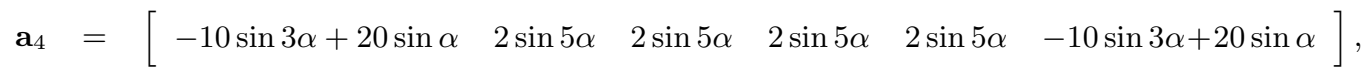

$$
\begin{aligned}
& \mathbf{a}_{5}=\left[\begin{array}{lllll}
4 \sin 4 \alpha & 0 & 0 & 0 & 4 \sin 4 \alpha
\end{array}\right], \\
& \mathbf{a}_{6}=\left[\begin{array}{llll}
15 \sin \alpha-12 \sin 3 \alpha & -5 \sin 3 \alpha & -5 \sin 3 \alpha & 15 \sin \alpha-12 \sin 3 \alpha
\end{array}\right], \\
& {\left[\begin{array}{lll}
\mathbf{a}_{7} & \mathbf{a}_{8} & \mathbf{a}_{9}
\end{array}\right]=\left[\begin{array}{llllll}
0 & 0 & 0 & 56 \sin \alpha & 56 \sin \alpha & 0
\end{array}\right] .}
\end{aligned}
$$


10. For $N=10$, we have $\alpha \in\left[\frac{11 \pi}{20}, \frac{5 \pi}{9}\right)$ and a non-trivial nonnegative solution is given by

$$
\begin{aligned}
& \mathbf{y}^{T}=\left[\begin{array}{lllllllllll}
\mathbf{a}_{0} & \mathbf{a}_{1} & \mathbf{a}_{2} & \mathbf{a}_{3} & \mathbf{a}_{4} & \mathbf{a}_{5} & \mathbf{a}_{6} & \mathbf{a}_{7} & \mathbf{a}_{8} & \mathbf{a}_{9} & \mathbf{a}_{10}
\end{array}\right] \text {, where } \\
& \mathbf{a}_{0}=\left[\begin{array}{lllllllllll}
504 & 0 & 0 & 0 & 0 & 4 \cos 10 \alpha & 0 & 0 & 0 & 0 & 504
\end{array}\right] \\
& \mathbf{a}_{1}=\left[\begin{array}{llllllllll}
-252 \cos \alpha & 0 & 0 & 0 & -2 \cos 9 \alpha & -2 \cos 9 \alpha & 0 & 0 & 0 & -252 \cos \alpha
\end{array}\right], \\
& \mathbf{a}_{2}=\left[\begin{array}{lllllllll}
70 & 0 & 0 & 0 & 2 \cos 8 \alpha & 0 & 0 & 0 & 70
\end{array}\right] \\
& \mathbf{a}_{3}=\left[\begin{array}{llllllll}
21 \cos 3 \alpha & 0 & \cos 7 \alpha & 0 & 0 & \cos 7 \alpha & 0 & 21 \cos 3 \alpha
\end{array}\right], \\
& \mathbf{a}_{4}=\left[\begin{array}{lllllll}
-30 \cos 2 \alpha & 0 & -2 \cos 6 \alpha & 0 & -2 \cos 6 \alpha & 0 & -30 \cos 2 \alpha
\end{array}\right], \\
& \mathbf{a}_{5}=\left[\begin{array}{llllll}
-4 \cos 5 \alpha & 0 & 0 & 0 & 0 & -4 \cos 5 \alpha
\end{array}\right] \\
& \mathbf{a}_{6}=\left[\begin{array}{lllll}
12 \cos 4 \alpha+15 & 0 & 5 \cos 4 \alpha & 0 & 12 \cos 4 \alpha+15
\end{array}\right],
\end{aligned}
$$

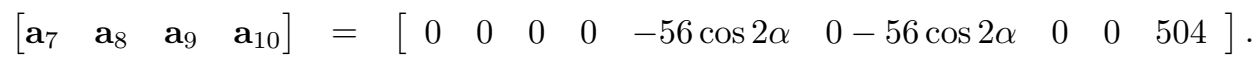

5. Final remark. It would be nice to affirm the sufficiency of the Conjecture 1.4 for $N>10$. Ideally, one can describe a non-trivial nonnegative solution of the linear system for every positive integer $N$. One may also consider finding an existence proof. In this connection, we have the following proposition. We will continue to use the notation $C_{\alpha, N}$ and consider the reduced system $C_{\alpha, N} \mathbf{y}=0$.

Proposition 5.1. Suppose $\alpha \in\left[\frac{\pi}{2}+\frac{\pi}{2 N}, \pi\right]$. The following conditions are equivalent.

(a) The system $C_{\alpha, N} \mathbf{y}=0$ has no non-trivial nonnegative solution.

(b) There is a complex vector $\mathbf{u}=\left(\xi_{0}, \ldots, \xi_{N}\right)$ with all entries having positive real parts such that all the entries of $\mathbf{u} C_{\alpha, N}$ has positive real parts.

Proof. We convert the system $C_{\alpha, N} \mathbf{y}=0$ to a real linear system

$$
\tilde{C}_{\alpha, N} \mathbf{y}=0, \quad \text { where } \tilde{C}_{\alpha, N}=\left[\begin{array}{l}
\Re\left(C_{\alpha, N}\right) \\
\Im\left(C_{\alpha, N}\right)
\end{array}\right]
$$

By Farkas lemma, for example see [1, Section 5.8], the system (5.10) has no non-trivial nonnegative solution if and only if there is a real vector $\mathbf{v}=\left(a_{0}, \ldots, a_{N}, b_{0}, \ldots, b_{N}\right)$ such that $\mathbf{v} \tilde{C}_{\alpha, N}$ is a positive vector, i.e., all entries are positive. Note that $a_{0}, a_{1}, \ldots, a_{N}$ appear in $\mathbf{v} \tilde{C}_{\alpha, N}$ as the $j$ th entries for $j=1,1+(N+1), 1+$ $(N+1)+N, 1+(N+1)+N+(N-1), \ldots,(N+1)(N+2) / 2$. So, $a_{0}, \ldots, a_{N}>0$ if the said vector v exists. Set $\xi_{j}=a_{j}-i b_{j}$ for $j=0, \ldots, N$. Then the system (5.10) has no non-trivial nonnegative solution if and only if condition (b) holds.

Acknowledgments. The authors would like to thank Runyao Duan, Cheng Guo, Yinan Li, and Weiyan $\mathrm{Yu}$ for some helpful correspondence and discussion. The authors would also like to express gratitude to the ELA referee who gave useful comments to improve this paper. 
Electronic Journal of Linear Algebra, ISSN 1081-3810

\section{REFERENCES}

[1] S. Boyd and L. Vandenberghe. Convex Optimization. Cambridge University Press, Cambridge, 2004.

[2] M.D. Choi. Completely positive linear maps on complex matrices. Linear Algebra Appl., 10(3):285-290, 1975.

[3] R. Duan, C. Guo, C.K. Li, and Y. Li. Parallel distinguishability of quantum operations. 2016 IEEE International Symposium on Information Theory (ISIT), 2259-2263, 2016.

[4] K. Kraus. States, Effects, and Operations: Fundamental Notions of Quantum Theory. Lectures in Mathematical Physics at the University of Texas at Austin, Vol. 190, Springer, Berlin, 1983.

[5] M.A. Neilsen and I.L. Chuang. Quantum Computation and Quantum Information. Cambridge University Press, New York, 2000.

[6] J. Watrous. Distinguishing quantum operations having few Kraus operators. Quant. Inf. Comput., 8(8):819-833, 2008. 